\title{
Understanding the role of adenosine receptors in the myofibroblast transformation in Peyronie's disease
}

Marta Mateus, MSc ${ }^{1}$, Marcus M. Ilg, MSc ${ }^{1}$, William J. Stebbeds, $\mathrm{PhD}^{2}$, Nim Christopher, MPhil, FRCS ${ }^{3}$, Asif Muneer, MD, FRCS (Urol) $)^{3,4}$, David J. Ralph, BSc, MS, FRCS (Urol) ${ }^{3}$ and Selim Cellek, MD, PhD, FBPhS ${ }^{*}$

${ }^{1}$ Anglia Ruskin University, Faculty of Medical Science, Chelmsford, UK

${ }^{2}$ Cranfield University, Bedfordshire, UK

${ }^{3}$ University College London Hospital, London, UK

${ }^{4}$ NIHR Biomedical Research Centre UCLH, London, UK

*Corresponding author (selim.cellek@anglia.ac.uk)

Conflict of Interest: None

Acknowledgement: The authors are indebted to the patients without whose tissue donations this study would not be possible. The authors also thank Drs Amr Raheem, Marco Spilotros, Arie Parnham and Giulio Garaffa for facilitating the tissue collections. This study was funded by European Society for Sexual Medicine. Asif Muneer is supported by the NIHR Biomedical Research Centre UCLH. 


\begin{abstract}
Background: Peyronie's disease (PD) is a chronic fibrotic disease of the penis affecting a significant number of men worldwide without effective medical treatments. Myofibroblasts are pivotal in the pathogenesis of PD. Adenosine and adenosine receptors have been suggested to be involved in the pathophysiology of fibrosis.

Aim: To understand the role of adenosine receptors in myofibroblast transformation in PD.

Methods: Fibroblasts were isolated from the non-PD tunica albuginea (TA) tissue and PD plaque tissue and were transformed into myofibroblasts using transforming growth factor $\beta 1$ (TGF- $\beta 1$ ). Quantification of alpha smooth muscle actin ( $\alpha-S M A)$ and adenosine receptors (ADORA1, ADORA2A, ADORA2B and ADORA3) was performed using immunocytochemistry (ICC), In-Cell ELISA (ICE) and real-time RT-PCR (RT-qPCR). The effect of various adenosine receptor agonists or antagonists on TGF- $\beta 1$-induced myofibroblast transformation was measured using ICE.

Outcomes: Expression of adenosine receptors in myofibroblasts obtained from human TA and the effect of adenosine receptor ligands on myofibroblast transformation.

Results: ICC, ICE and RT-qPCR experiments showed that the protein and mRNA levels of $\alpha-S M A$ in nonPD TA cells and PD plaque-derived cells were significantly higher in cells exposed to TGF- $\beta 1$ than those not treated with TGF- $\beta 1$. Two of four adenosine receptors (ADORA1 and ADORA2B) were found to be expressed in both cell populations. Among various adenosine receptor agonists/antagonist investigated, only ADORA2B agonist, BAY60-6583, significantly inhibited myofibroblast transformation in a concentration-dependent manner when applied simultaneously with TGF- $\beta 1\left(I C_{50}=30 \mu \mathrm{M}\right)$.

Clinical Translation: ADORA2B antagonists may be clinically efficacious in early stage PD.

Strengths \& Limitations: The strength of this study is the use of primary fibroblasts from human TA. Limitation of the study is the high concentrations of the ligands used.

Conclusions: The effect of an ADORA2B agonist on TGF- $\beta 1$-induced myofibroblast transformation shows a novel potential therapeutic target for PD if applied during early, non-stable phase of PD.
\end{abstract}

KEYWORDS: fibrosis, transforming growth factor, anti-fibrotic therapies, fibroblast, cell culture 


\section{INTRODUCTION}

Fibrosis can be described as excessive development of fibrous connective tissue, which can occur in various tissue types and organs (e.g. kidney, lung, skin and liver). At a cellular level tissue resident quiescent fibroblasts and other cells such as endothelial, epithelial cells, and fibrocytes can differentiate into myofibroblasts which have a crucial role in fibrosis characterised by increased proliferation, increased extracellular matrix (ECM) protein production and contraction ${ }^{1,2}$. Moreover, their persistence (i.e. failure to undergo apoptosis) and proliferation has been suggested to be one of the hallmarks of chronic fibrosis ${ }^{3,4}$.

Peyronie's disease (PD) is a fibrotic disorder characterised by the formation of plaques within the tunica albuginea (TA) of the penis ${ }^{5}$. Although its aetiology is still poorly understood, microvascular trauma has been postulated as the initiating factor ${ }^{6}$. This fibrotic disorder is also characterised by the expression of several cytokines and growth factors, fibrin deposition and myofibroblast differentiation ${ }^{7}$. In PD, the myofibroblast activity is increased, resulting in increased ECM protein production and eventual plaque formation ${ }^{7-11}$ suggesting a pivotal role for myofibroblasts in the pathophysiology of PD.

Inhibition of differentiation of quiescent fibroblasts to pro-fibrotic myofibroblasts has been suggested as a therapeutic approach for fibrosis ${ }^{4}$. Accordingly, we have been investigating potential molecular targets that may be involved in myofibroblast differentiation and small molecule compounds that may inhibit this process.

One of such target that is suggested in pathophysiology of fibrosis is adenosine and its receptors. Adenosine is a ubiquitous purine nucleoside released from cells and tissues under conditions of stress or injury and is generated intracellularly and extracellularly from adenine nucleotides which are then dephosphorylated to adenosine. CD39 and CD73 are two cell surface molecules responsible for catalysing the dephosphorylation of adenine nucleotides to adenosine in the extracellular space ${ }^{12,13}$. Adenosine regulates its effects on tissue regeneration and repair via the interaction with a family of G-protein coupled receptors: adenosine receptor $A 1$ (ADORA1), adenosine receptor $A 2 A$ ( $A D O R A 2 A)$, adenosine receptor A2B (ADORA2B) and adenosine receptor A3 (ADORA3) ${ }^{14}$.

Several studies have shown that adenosine receptors play different roles in acute and chronic injuries. In acute tissue injury, adenosine has been shown to be beneficial, as it is responsible for tissue protection and anti-inflammatory responses ${ }^{13}$ (e.g. promotion of barrier function and wound healing) in several organs, including kidney ${ }^{15}$, lung ${ }^{16}$, heart ${ }^{17}$ and liver $^{18}$. In contrast to acute states, increased levels of adenosine have been associated with the progression of chronic tissue injuries. In these settings, adenosine has been suggested to promote fibrosis in several organs, such as the heart ${ }^{19}$, skin ${ }^{20}$, liver ${ }^{21}$, lung ${ }^{22}$, penis ${ }^{23}$ and kidney ${ }^{24}$. The adenosine receptors play different roles in the pathogenesis of fibrosis depending on the tissue subtype involved; however, the effects of adenosine are mainly regulated by ADORA2A and $A D O R A 2 B^{16}$. The characterisation of these receptors has been investigated in other fibrotic disorders; however, no characterisation of these receptors has been carried out in Peyronie's disease. By 
understanding how adenosine receptors may regulate the response to injury in this specific tissue and by looking at the myofibroblast transformation process, it may provide new insights into the pathophysiology of fibrosis in general and in PD. Furthermore, by targeting the respective pathway and by investigating the effect of selective agonist and antagonist compounds, it may enable avenues to identify potential targets for the treatment of PD and other fibrotic disorders and enhance the resolution of the injury or halt the progression of fibrosis in PD.

The aim of this study was to understand the involvement of adenosine receptors in the myofibroblast differentiation in PD by characterising the myofibroblast transformation process in tunica albuginea-derived fibroblasts to identify potential, novel targets for anti-fibrotic therapies. 


\section{METHODS}

\section{Sample acquisition}

Tunica albuginea (TA) tissue samples were acquired from patients undergoing corrective surgery for PD (to be referred to as PD plaque tissue) or invasive penile cancer (to be referred to as non-PD TA tissue) at University College London Hospital (UCLH), UK. Patients aged between 18 and 75, listed for surgical treatment of PD or penile cancer at $\mathrm{UCLH}$, able to understand the patient information sheet and to give consent were included in this study. Ethical approval was obtained by local independent research ethics committees (East of England Essex [12-EE-0170] and North of Scotland [15-NS-0051]).

The non-PD TA tissue was obtained from penis of patients with penile cancer $(\mathrm{N}=3$; average age $=72 \pm 10)$. The TA tissue was removed from the proximal side away from the tumour and the tumour had negative margins on histology examination.

The PD TA tissue was obtained from penis of patients with chronic PD $(\mathrm{N}=3$; average age $=61 \pm 6)$. The tissue used in this group was the plaque tissue that was surgically removed and would have otherwise been discarded.

\section{Isolation of fibroblasts from TA tissue}

Tissue samples were dissected into small pieces, submerged in culture media in 6 well plates and incubated at $37^{\circ} \mathrm{C}, 5 \% \mathrm{CO}_{2}$ in a humidified atmosphere for 5-7 days. This method is known as "explant technique" by which pure fibroblast cultures can be obtained ${ }^{25}$. Tissues were removed from the 6 well plates, once cells were observed growing out of the tissue. Cells were then washed three times with PBS and fresh, warm medium was added to each well. Cells were incubated at $37^{\circ} \mathrm{C}$ until they reached $50-70 \%$ confluence, after which old medium was removed and cells were washed with PBS. Cells were detached with $0.25 \%$ trypsin/EDTA (TE; GIBCO, Invitrogen, UK) and neutralised with culture media. The cell suspension was transferred to T75 flasks (NUNC, Thermo Scientific, UK) and the cells were propagated and maintained up to passage 10 .

\section{Real-time RT-PCR (RT-qPCR)}

Cells were seeded onto 6 well plates (Nunc, Fisher Scientific, UK) at $1.0 \times 10^{5}$ cells/well and incubated in DMEM-F12 medium (GIBCO, Invitrogen, UK) supplemented with 10\% FCS (Fisher Scientific, UK) and 1\% penicillin-streptomycin (GIBCO, Invitrogen, UK). After overnight incubation, fibroblasts were incubated with or without TGF- $\beta 1$ (Sigma-Aldrich, UK; $10 \mathrm{ng} / \mathrm{ml}$ ) for 72 hours. Total RNA was extracted from cells using the RNeasy Mini Kit (QIAGEN, UK) according to the manufacturer's instructions and a DNAse digestion step was included to eliminate genomic DNA contamination (RNAse-free DNAse set, QIAGEN, UK). cDNA was generated from $500 \mathrm{ng}$ of RNA by reverse transcription using the High Capacity cDNA Reverse Transcription kit (Applied Biosystems, UK), according to manufacturer's instructions. Real-time PCR was 
performed using the QuantiTect® SYBR® Green PCR kit (QIAGEN, UK), following the manufacturer's instructions and the CFX Connect Real-time PCR detection system (Bio-Rad, UK). Gene-specific primer pairs for genes encoding a-SMA (NM_001613, 98 bp) were purchased from Sigma-Aldrich, UK and ADORA1 (NM_000674.2, 96 bp), ADORA2A (NM_000675.5, 93 bp), ADORA2B (NM_000676.2, 117 bp) and ADORA3 (NM_000677.3, $139 \mathrm{bp}$ ) were purchased from Primerdesign, UK. The primers for EIF4A2 (NM_001967, 87 bp), TOP1 (NM_003286, 89 bp), vimentin (NM_003380) and desmin (NM_001927) were purchased from QIAGEN, UK. PCR reactions were carried out by performing an initial activation step for 15 minutes at $95^{\circ} \mathrm{C}$, followed by 40 cycles at $94^{\circ} \mathrm{C}$ for 15 seconds, $55^{\circ} \mathrm{C}$ for 30 seconds and $72^{\circ} \mathrm{C}$ for 30 seconds. All samples and negative controls (no template control and no reverse transcriptase enzyme control) were run in triplicate and in addition, a melting curve was generated at the end of the PCR cycle to ensure amplification of a single product. Relative mRNA levels were calculated using the $2^{-\Delta \Delta C t}$ method $^{26}$ and obtained as fold-change of the target gene in the test samples relative to the calibrator sample and normalised to the expression of the reference genes (EIF4A2 and TOP1).

\section{Immunocytochemistry (ICC)}

Fibroblasts obtained from tunica albuginea tissue samples were seeded onto coverslips (Fisher Scientific, UK) at $2.5 \times 10^{5}$ cells/well and were incubated with or without TGF- $\beta 1(10 \mathrm{ng} / \mathrm{ml})$ for 72 hours. After incubation, cells were washed with PBS (GIBCO, Invitrogen, UK) and fixed in ice-cold methanol $\left(-25^{\circ} \mathrm{C}\right.$; Fisher Scientific, UK) for 10 seconds. Cells were then washed with PBS and 10\% donkey serum (EMDMillipore, UK) in PBS was added for 1 hour at room temperature. Blocking solution was then replaced by primary antibody solution (1:1,000 dilution of mouse monoclonal anti-a-SMA antibody, Sigma-Aldrich, UK; 1:1,000 dilution of mouse monoclonal vimentin, Abcam, UK; or 1:100 mouse monoclonal desmin, Abcam, UK) diluted in PBS for 2 hours at room temperature in a humidified chamber. Cells were washed three times with PBS, after which, they were incubated with the donkey anti-mouse secondary antibody conjugated with fluorescein dye (Millipore, UK) at 1:250 dilution in PBS for 2 hours at room temperature in a humidified chamber in the dark. After the incubation period, the secondary antibody solution was washed three times with PBS and the coverslips were mounted in a glass microscope slide with cells facing down with VECTASHIELD® mounting medium with propidium iodide (PI; Vector Laboratories, UK). Cells were observed and images were captured using the Zeiss confocal microscope (LSM 510; Carl Zeiss, UK). The fluorescence images were taken in at least three random areas in each coverslip from each cell line acquired from three different patients (per group) (PD and non-PD). The number of a-SMA-positive cells was determined by counting the number of $\alpha$-SMA-positive cells in three random fields per coverslip and the total number of cells was also determined by counting the number of nuclei stained.

\section{In-Cell ELISA (ICE)}

The expression of $\alpha-S M A$ protein was quantified in 96 well plates using a technique called "In-Cell ELISA" (ICE) which involves fixation of the cells on the plate surface, permeabilisation of the cells, staining of the proteins with an antibody-ligated with infrared dyes and visualising the infrared-red dyes using an infra-red 
imaging system ${ }^{27}$. Cells were seeded onto 96 well optical flat bottom black microplates (Nunc, Fisher Scientific, UK) at $5.0 \times 10^{3}$ cells /well and left overnight at $37^{\circ} \mathrm{C}, 5 \% \mathrm{CO}_{2}$ in a humidified incubator. Media was replaced with fresh media with and without agonists and antagonists of adenosine receptors (all purchased from TOCRIS, UK) in co-incubation with or without TGF- $\beta 1$ (5 ng/ml) for 72 hours. N6Cyclopentyladenosine (CPA) and BAY 60-6583 were used as ADORA1 and ADORA2B agonists, respectively. SLV 320 and MRS 1754 were used as ADORA1 and ADORA2B antagonists, respectively. The compounds were dissolved in $100 \%$ DMSO to $100 \mathrm{mM}$ stock concentration and further diluted to 100 $\mu \mathrm{M}$ final assay concentration. After incubation, media was removed, and cells were fixed with $4 \%$ paraformaldehyde for 20 minutes at room temperature. Fixing solution was then removed and cells were washed three times with $0.1 \%$ Triton X-100 in PBS. A $5 \%$ donkey serum plus $0.1 \%$ Triton X-100 in PBS solution was incubated with cells for blocking and permeabilisation for 90 minutes at room temperature. After removing the buffer, a primary antibody solution diluted in PBS (1:3,000 dilution of mouse monoclonal anti-a-SMA antibody, 1:500 dilution of rabbit monoclonal anti-adenosine A1 receptor or 1:500 dilution of rabbit polyclonal anti-adenosine $\mathrm{A} 2 \mathrm{~B}$ receptor) was added and incubated for 2 hours at room temperature. Cells were then washed with $0.1 \%$ Tween 20 in PBS three times and a solution containing both the secondary antibody (donkey anti-mouse or donkey anti-rabbit at 1:500 which emits at $800 \mathrm{~nm}$; IRdye $800 \mathrm{CW}$; Li-Cor, UK) and a nuclear counterstain at 1:1,000 that emits at $700 \mathrm{~nm}$ (DRAQ5, Biostatus, UK) diluted in PBS was incubated for 1 hour at room temperature in the dark. Cells were washed three times with $0.1 \%$ Tween 20 in PBS and the plate was scanned using an infrared imaging system (Odyssey® CLx imager, Li-Cor, UK) at both $700 \mathrm{~nm}$ and $800 \mathrm{~nm}$ wavelengths.

\section{Statistical analysis}

Data analyses and graphs were plotted using Microsoft ${ }^{\circledR}$ Excel 2013 and presented as mean \pm standard error of the mean (SEM) since the data was normally distributed according to Shapiro-Wilks test. Statistical differences were determined by Student's t-test for unpaired means (two-sided) with a $P$ value less than 0.05 considered statistically significant. All experiments were carried out in triplicate using samples from at least 3 patients $(\mathrm{N}=3)$. 


\section{RESULTS}

\section{Characterisation of cells isolated from non-PD TA tissue and PD plaque tissue}

In order to ascertain that the cells we have isolated from human TA were fibroblasts, we investigated the expression of vimentin and desmin since fibroblasts are known to be vimentin-positive and desminnegative ${ }^{28}$. Vimentin was expressed at both protein and mRNA levels in cells obtained from PD plaque tissue and non-PD TA tissues. Treatment of the cells with TGF- $\beta 1$ did not alter the expression of vimentin (Figure 1).

The cells established from non-PD TA tissue and PD plaque tissue were desmin-negative; this was not affected by TGF- $\beta 1$ treatment (Figure 1). These results confirm that the cells isolated from TA were indeed fibroblasts.

\section{Cells derived from non-PD TA tissue and PD plaque tissue express $\alpha-S M A$}

Myofibroblasts are known to be vimentin-positive, desmin-negative and $\alpha$-SMA-positive ${ }^{29}$. The expression of $\alpha-S M A$ was investigated in cells isolated from non-PD TA tissue and PD plaque tissue. The mRNA levels of $\alpha-S M A$ significantly increased in both cell populations when exposed to TGF- $\beta 1$ for 72 hours (Figure 2E). In addition, $\alpha$-SMA positive cells were observed in both cell populations in the presence of TGF- $\beta 1$ (Figure 2-C and 2-D); whereas, in the absence of TGF- $\beta 1$, the presence of $\alpha-S M A$ positive cells was rare or non-existent in cells established from non-PD TA tissue and PD plaque tissue (Figure 2-A and 2-B). The number of $\alpha$-SMA positive cells in each cell population exposed to TGF- $\beta 1$ was significantly higher compared to untreated cells (Figure 2-F). In order to quantify the protein levels in an objective and higher throughput manner, the ICE method was used to test the effect of TGF- $\beta 1$ on non-PD TA cells and PD plaque-derived cells. Similar to the immunocytochemistry results, treatment with TGF- $\beta 1$ elicited a significant increase in expression of $\alpha$-SMA protein in cells obtained from either non-PD TA or PD plaque tissues (Figure 3).

\section{Expression of adenosine receptors in non-PD TA cells and PD plaque-derived cells}

The expression of four adenosine receptors (ADORA1, ADORA2A, ADORA2B and ADORA3) was investigated in cells derived from non-PD TA tissue and PD plaque tissue. Both cell populations expressed low levels of ADORA2A and ADORA3 (Figure 4); whereas, ADORA2B was expressed in both cell groups isolated from healthy and fibrotic tissues and ADORA1 was expressed in cells derived from PD plaque tissue. ADORA1 and ADORA2B were significantly higher in cells established from PD plaque tissue than from non-PD TA tissue (Figure 4).

\section{Effect of TGF- $\beta 1$ in ADORA1 and ADORA2B in cells derived from non-PD TA tissue and PD plaque} tissue 
The transcriptional levels of ADORA1 were significantly reduced in cells treated with TGF- $\beta 1$ compared to untreated cells that were derived from non-PD TA tissue and PD plaque tissue. However, no statistically significant difference was observed between non-PD TA cells and PD plaque-derived cells (Figure 5-A). Contrary to mRNA levels, the protein levels of ADORA1 were significantly increased when exposed to TGF$\beta 1$ in cells isolated from non-PD TA tissue and PD plaque tissue (Figure 5-B).

The mRNA levels of ADORA2B were significantly decreased in cells exposed to TGF- $\beta 1$ compared to cells not exposed to TGF- $\beta 1$ (Figure 6-A). Although, no statistically significant difference was observed in the protein levels of ADORA2B when treated with TGF- $\beta 1$, significance was achieved between non-PD TA cells and PD plaque-derived cells (Figure 6-B).

\section{Effect of ADORA1 and ADORA2B agonist and antagonist on TGF- $\beta 1$-induced myofibroblast transformation}

To further investigate the change of ADORA1 and ADORA2B expression and their ability to inhibit myofibroblast transformation, agonists and antagonists were used. N6-Cyclopentyladenosine (CPA) and BAY 60-6583 were used as ADORA1 and ADORA2B agonists, respectively. On the other hand, SLV 320 and MRS 1754 were used as ADORA1 and ADORA2B antagonists, respectively.

ADORA1 agonist (CPA) showed inhibition of TGF- $\beta 1$-induced myofibroblast transformation only at $100 \mu \mathrm{M}$ in either cell populations. At the same high concentration, the cell viability was also reduced (Figure 7-A). ADORA2B agonist (BAY60-6583) significantly inhibited TGF- $\beta 1$-induced myofibroblast transformation in a concentration dependent manner in both non-PD TA cells and PD plaque-derived cells $\left(\mathrm{IC}_{50}=30 \mu \mathrm{M}\right)$ (Figure 7-B, 7-E). The compound did not affect cell viability at any concentration tested in TGF- $\beta 1$-induced myofibroblasts (Figure 7-B) or non-stimulated fibroblasts (Supplementary Figure 1).

ADORA1 antagonist, SLV 320, inhibited TGF- $\beta 1$-induced myofibroblast transformation only at $100 \mu \mathrm{M}$; without affecting the cell viability (Figure 7-C). The ADORA2B antagonist, MRS 1754, also failed to inhibit TGF- $\beta 1$-induced myofibroblast transformation in either cell population significantly; the inhibition that was observed at only high concentrations was due to decreased cell viability (Figure 7-D). 


\section{DISCUSSION}

Although adenosine and its receptors have been studied in other fibrotic diseases, there is a lack of studies reporting the characterisation and effects of adenosine receptors in PD. Therefore, the aim of this study was to investigate the role of adenosine receptors in myofibroblast transformation in PD.

First, the cells isolated from non-PD TA tissue and PD plaque tissue were characterised. The results showed that both cell groups were positive for vimentin and negative for desmin suggesting that these cells were fibroblasts. It was also observed that treatment with TGF- $\beta 1$ did not alter the expression of these specific markers, but significantly increased the expression of $\alpha$-SMA confirming myofibroblast phenotype ${ }^{29}$. Myofibroblasts have a crucial role in pathophysiology of fibrosis, as these cells are not only responsible for contraction but also for production of several cytokines and abundant ECM proteins. Myofibroblast transformation is well established in the literature as being an important contributor in the pathophysiology of several fibrotic disorders including PD. A key feature of myofibroblasts is their $\alpha$-SMA expression, which can be targeted to investigate the myofibroblast differentiation. Both mRNA and protein levels of $\alpha$-SMA were assessed by exposing cells derived from non-PD TA tissue and PD plaque tissue to TGF- $\beta 1$. In both cell groups, a significant increase of $\alpha$-SMA mRNA levels was observed when treated with TGF- $\beta 1$. The effect of TGF- $\beta 1$ on mRNA levels was mirrored by $\alpha$-SMA protein expression, that is, both cell populations showed an increase in $\alpha-S M A$ positive cells in the presence of TGF- $\beta 1$. These results are supported by previously published reports that have shown that both $\alpha$-SMA mRNA and protein levels increase in the presence of TGF- $\beta 1$ in fibroblasts obtained from PD tissue ${ }^{10,11}$.

Interestingly, a-SMA-positive cell numbers and a-SMA mRNA and protein levels in unstimulated cells obtained from PD tissue were similar to those from TA tissue suggesting that basal myofibroblast numbers were similar in cultures derived from the two tissue types. Although we did not aim to investigate specifically whether PD tissue would yield more myofibroblasts than TA tissue, this was a surprising and unexpected finding. We believe that this could be as a result of the choice of method we employed to isolate cells from the tissue samples. During the explantation of the tissue and subsequent cell passaging, more fibroblasts than myofibroblasts might have migrated from the tissue or myofibroblasts might have reverted in their phenotype during cell passaging. Another possibility is that the PD cells could have already been exposed to TGF- $\beta 1$ in the tissue during the formation of the plaque; they may have developed resistance to TGF- $\beta 1$ hence their lower responsiveness to TGF- $\beta 1$ than the TA cells. Nevertheless, our results confirmed that both tissue types yielded sufficient number of fibroblasts which can be transformed to myofibroblasts using TGF- $\beta 1$ and can be further utilised in myofibroblast transformation assay.

Adenosine receptors have been associated with the progression of chronic tissue injuries when the levels of adenosine are increased, suggesting that adenosine may promote fibrosis. To the best of the author's knowledge, this is the first time that the expression of adenosine receptors has been investigated in cells 
derived from non-PD TA tissue and PD plaque tissue. In order to demonstrate the involvement of adenosine receptors in myofibroblast transformation in PD, the expression of these receptors was investigated in cells isolated from non-PD TA tissue and PD plaque tissue. Among the four receptors, the expression of ADORA1 and ADORA2B was significantly higher in PD plaque-derived cells than in non-PD TA cells; however, this increase was dramatically higher in ADORA1 transcripts levels. The expression of ADORA2A and ADORA3 were below the detection limits, therefore these two receptors were not studied further. Zhong et al. (2005) 30 found that primary human lung fibroblasts expressed high mRNA levels of ADORA2B and the expression of this receptor at protein levels was confirmed by immunofluorescence. These authors also showed that the activation of the ADORA2B by adenosine promoted myofibroblast transformation. In our study, the effect of TGF- $\beta 1$ on mRNA levels of both adenosine receptors was also assessed, leading to a significant decrease of ADORA1 and ADORA2B mRNA levels in both cell groups. Even though the mRNA levels were significantly decreased, it may not represent a biological change in the transcript levels, as according to MIQE guidelines, to show biological changes a 2-fold (recommended cut-off value) increase or decrease should be observed ${ }^{31}$. The protein levels of ADORA1 and ADORA2B in both cell populations was assessed using the ICE assay, showing that a significant increase of ADORA1 was observed in cells treated with TGF- $\beta 1$; however, no difference was observed between non-PD TA cells and PD plaquederived cells.

In addition, Wen et al. (2010)23 showed that primary corpus cavernosal fibroblasts from mice expressed ADORA2B, which was suggested to be responsible for adenosine-mediated penile fibrosis. Furthermore, two other studies have also shown that deaminase-deficient mice had an increment of adenosine levels and ADORA2B activation in the penis, suggesting an essential mechanism for the progression of priapism in these mice ${ }^{23,32}$.

Receptor expression results therefore suggest that ADORA1 and ADORA2B were expressed in both fibroblasts and myofibroblasts derived from TA. It would be worth investigating in the future how these two receptors and their downstream pathways may interact in physiological and pathological conditions.

The role of ADORA1 and ADORA2B was assessed in TGF- $\beta 1$-induced myofibroblast transformation using ADORA1 and ADORA2B agonists and antagonists. CPA and BAY 60-6583 were utilised as ADORA1 and ADORA2B agonists, respectively while SLV 320 and MRS 1754 were used as ADORA1 and ADORA2B antagonists, respectively. ADORA1 agonist, CPA showed an inhibitory effect on TGF- $\beta 1$-induced myofibroblast transformation only at high concentrations while affecting the cell viability; therefore, we conclude that the inhibitory effect is most probably due to cytotoxicity at the high concentrations. ADORA2B agonist BAY60-6583 significantly inhibited the transformation in a concentration-dependent manner with an $\mathrm{IC}_{50}$ value of $30 \mu \mathrm{M}$ and had no cytotoxicity either on fibroblasts or myofibroblasts. No inhibition of TGF$\beta 1$-induced myofibroblast transformation was observed with the ADORA1 antagonist (SLV320) and ADORA2B antagonist (MRS1754). We are particularly surprised to see no effect by ADORA2B antagonist since we would have expected inhibition of myofibroblast transformation with this compound. However, we 
have observed an inhibition by ADORA2B agonist suggesting that endogenous adenosine may have antifibrotic effect under these conditions which obviously warrants further research.

We conclude that among the four agonists/antagonists tested, ADORA2B agonist BAY60-6583 has proven to be the most successful one and further suggest that $A D O R A 2 B$ agonism may be potential therapeutic approach for PD. This is further supported by our finding of ADORA2B expression in cells derived from human TA with or without PD. It would be worthwhile testing this compound in animal models of Peyronie's disease.

To our knowledge, BAY60-6583 has not been progressed to clinical development and remains to be the only ADORA2B agonist that has been developed ${ }^{33,34}$. Further development of similar compounds would be beneficial in future fibrosis research.

One of the limitations of our study is being limited to in vitro findings. Although we have used primary fibroblasts isolated from fresh human tissue, there is always the risk of our findings not being able to be translated to in vivo. Similarly, the $\mathrm{IC}_{50}$ of BAY60-6583 is relatively high; it is unlikely that such a concentration can be reached at tissue level in an in vivo situation. Further work would be required to understand the pharmacology of ADORA2B agonists in PD. Another limitation is the choice of non-PD TA tissue being obtained from patients with penile cancer. Although we made sure that the tissue obtained was away from the tumour and the tumour margins were negative in histology examination, we cannot exclude the possibility of cancer genetics influencing the fibroblast phenotype.

\section{CONCLUSIONS}

Cell culture models from both non-PD TA tissue and PD plaque tissue were successfully established, with confirmed fibroblast identity and differences regarding TGF- $\beta 1$-induced myofibroblast transformation, mainly increased $\alpha$-SMA mRNA and protein levels in cells isolated from PD plaque tissue.

The expression of two adenosine receptors (ADORA1 and ADORA2B) was demonstrated in both cell groups in which the ADORA2B agonist BAY 60-6583 significantly inhibited TGF- $\beta 1$-induced myofibroblast transformation in the two cell types investigated.

Inhibition of differentiation of quiescent fibroblasts to pro-fibrotic myofibroblasts has been suggested as a therapeutic approach for fibrosis ${ }^{4}$. The effect of BAY $60-6583$ on TGF- $\beta 1$-induced myofibroblast transformation may suggest a novel potential therapeutic target for PD if applied during early, non-stable phase of PD and in other fibroproliferative diseases, although we did not test the compounds on cells derived from the acute phase of PD. We suggest that such compounds may be useful in early stages of the disease to prevent formation of the plaque. 


\section{REFERENCES}

1. Wynn, T. A. \& Ramalingam, T. R. Mechanisms of fibrosis: therapeutic translation for fibrotic disease. Nat Med 18, 1028-1040 (2012).

2. Krenning, G., Zeisberg, E. M. \& Kalluri, R. The origin of fibroblasts and mechanism of cardiac fibrosis. J Cell Physiol 225, 631-637 (2010).

3. Shirol, P. D. \& Shirol, D. D. Myofibroblasts in health and disease. Int J Oral Maxillofac Pathol 3, 23-27 (2012).

4. Bollong, M. J. et al. Small molecule-mediated inhibition of myofibroblast transdifferentiation for the treatment of fibrosis. Proc Natl Acad Sci 114, 4679-4684 (2017).

5. Garaffa, G., Trost, L. W., Serefoglu, E. C., Ralph, D. \& Hellstrom, W. J. G. Understanding the course of Peyronie's disease. Int J Clin Pract 67, 781-788 (2013).

6. Langston, J. P. \& Carson, C. C. Peyronie's disease: review and recent advances. Maturitas 78, 341-343 (2014).

7. Gelfand, R. A., Vernet, D., Kovanecz, I., Rajfer, J. \& Gonzalez-Cadavid, N. F. The transcriptional signatures of cells from the human Peyronie's disease plaque and the ability of these cells to generate a plaque in a rat model suggest potential therapeutic targets. J Sex Med 12, 313-327 (2015).

8. Jalkut, M., Gonzalez-Cadavid, N. \& Rajfer, J. New discoveries in the basic science understanding of Peyronie's disease. Curr Urol Rep 5, 478-484 (2004).

9. Gonzalez-Cadavid, N. \& Rajfer, J. Mechanisms of disease: new insights into the cellular and molecular pathology of Peyronie's disease. Nat Clin Pract Urol 2, 291-297 (2005).

10. Mulhall, J. P., Anderson, M. S., Lubrano, T. \& Shankey, T. V. Peyronie's disease cell culture models: phenotypic, genotypic and functional analyses. Int J Impot Res 14, 397-405 (2002).

11. Vernet, D. et al. Effect of nitric oxide on the differentiation of fibroblasts into myofibroblasts in the Peyronie's fibrotic plaque and in its rat model. Nitric Oxide 7, 262-276 (2002).

12. Cronstein, B. N. Adenosine receptors and fibrosis: a translational review. F1000 Biol Rep 3, 1-6 (2011).

13. Karmouty-Quintana, H., Xia, Y. \& Blackburn, M. R. Adenosine signaling during acute and chronic disease states. J Mol Med 91, 173-181 (2013).

14. Chen, J.-F., Eltzschig, H. K. \& Fredholm, B. B. Adenosine receptors as drug targets - what are the challenges? Nat Rev Drug Discov 12, 265-286 (2013).

15. Grenz, A. et al. The reno-vascular A2B adenosine receptor protects the kidney from ischemia. PLoS Med 5, 968-986 (2008).

16. Zhou, Y. et al. Distinct roles for the A2B adenosine receptor in acute and chronic stages of bleomycin-induced lung injury. J Immunol 186, 1097-1106 (2011).

17. Eckle, T. et al. Cardioprotection by Ecto-5'-Nucleotidase (CD73) and A2B adenosine receptors. Circulation 115, 1581-1590 (2007).

18. Day, Y.-J. et al. Protection from ischemic liver injury by activation of A2A adenosine receptors during reperfusion: inhibition of chemokine induction. Am J Physiol Gastrointest Liver Physiol 286, G285-G293 (2004). 
19. Toldo, S. et al. GS-6201, a selective blocker of the A2B adenosine receptor, attenuates cardiac remodeling after acute myocardial infarction in the mouse. J Pharmacol Exp Ther 343, 587-595 (2012).

20. Fernández, P. et al. Pharmacological blockade of A2A receptors prevents dermal fibrosis in a model of elevated tissue adenosine. Am J Pathol 172, 1675-1682 (2008).

21. Chan, E. S. L. et al. Adenosine $\mathrm{A}(2 \mathrm{~A})$ receptors play a role in the pathogenesis of hepatic cirrhosis. Br J Pharmacol 148, 1144-1155 (2006).

22. Sun, C.-X. et al. Role of A2B adenosine receptor signaling in adenosine-dependent pulmonary inflammation and injury. J Clin Invest 116, 2173-2182 (2006).

23. Wen, J. et al. Increased adenosine contributes to penile fibrosis, a dangerous feature of priapism, via A2B adenosine receptor signaling. FASEB J 24, 740-749 (2010).

24. Dai, Y. et al. A2B adenosine receptor-mediated induction of IL-6 promotes CKD. J Am Soc Nephrol 22, 890-901 (2011).

25. Cheung, H. S. An improved method of establishing human fibroblast cultures from explants. $J$ tissue Cult methods 6, 39-40 (1980).

26. Livak, K. J. \& Schmittgen, T. D. Analysis of relative gene expression data using real-time quantitative PCR and the 2(-Delta Delta C(T)) Method. Methods 25, 402-408 (2001).

27. Aguilar, H. N., Zielnik, B., Tracey, C. N. \& Mitchell, B. F. Quantification of rapid myosin regulatory light chain phosphorylation using high-throughput in-cell western assays: comparison to western immunoblots. PLoS One 5, e9965 (2010).

28. Sampson, N., Berger, P. \& Zenzmaier, C. Therapeutic targeting of redox signaling in myofibroblast differentiation and age-related fibrotic disease. Oxid Med Cell Longev 2012, 1-15 (2012).

29. Duffield, J. S. Cellular and molecular mechanisms in kidney fibrosis. J Clin Invest 124, 2299-2306 (2014).

30. Zhong, H., Belardinelli, L., Maa, T. \& Zeng, D. Synergy between A2B adenosine receptors and hypoxia in activating human lung fibroblasts. Am J Respir Cell Mol Biol 32, 2-8 (2005).

31. Bustin, S. A. et al. MIQE précis: Practical implementation of minimum standard guidelines for fluorescence-based quantitative real-time PCR experiments. BMC Mol Biol 11, 1-5 (2010).

32. $\mathrm{Mi}, \mathrm{T}$. et al. Excess adenosine in murine penile erectile tissues contributes to priapism via A2B adenosine receptor signaling. J Clin Invest 118, 1491-1501 (2008).

33. Gao, Z.-G. \& Jacobson, K. A. Emerging adenosine receptor agonists-an update. Expert Opin Emerg Drugs 16, 597-602 (2011).

34. Hinz, S., Lacher, S. K., Seibt, B. F. \& Müller, C. E. BAY60-6583 acts as a partial agonist at adenosine A2B receptors. J Pharmacol Exp Ther 349, 427-36 (2014). 


\section{FIGURE LEGENDS}

Figure 1: Vimentin and desmin quantification in cells derived from patients with or without Peyronie's disease. Fibroblasts were exposed to TGF- $\beta 1(10 \mathrm{ng} / \mathrm{ml})$ for 72 hours. A, B, C\&D: Representative images of $(A)$ vimentin stained non-PD TA-derived cells exposed to control conditions; (B) desmin stained non-PD TA-derived cells exposed to control conditions; (C) vimentin stained non-PD TAderived cells exposed to TGF- $\beta 1$; (D) desmin stained non-PD TA-derived cells exposed to TGF- $\beta 1$. Images were captured at 200x magnification. Scale bars represent $50 \mu \mathrm{m}$. (E) Quantification of vimentin/desmin expression non-PD TA-derived cells using ICE. (F) Quantification of vimentin/desmin expression PD TAderived cells using ICE. Data points were plotted as mean \pm SEM, $N=3$ patients for each group. (G) Representative image for desmin staining of rhabdomyosarcoma cell line RD. (H) Quantification of vimentin mRNA expression of non-PD and PD TA-derived cells using GPCR. (I) Quantification of desmin mRNA expression of non-PD and PD TA-derived cells using qPCR. Fibroblasts were exposed to TGF- $\beta 1$ (10 $\mathrm{ng} / \mathrm{ml}$ ) for 72 hours before RNA isolation. Data points were plotted as mean $\pm \mathrm{SEM}, \mathrm{N}=3$ patients for each group. n.d.=not detectable.

Figure 1: Expression of $\alpha$-SMA in cells established from non-PD TA tissue and PD plaque tissue. (A) Representative illustrations of a-SMA staining in untreated non-PD TA cells, (B) Representative illustrations of $\alpha$-SMA staining in untreated PD plaque-derived cells, (C) Representative illustrations of $\alpha$ SMA staining in non-PD TA cells treated with TGF- $\beta 1$, (D) Representative illustrations of $\alpha-S M A$ staining in PD plaque-derived cells treated with TGF- $\beta 1$. The nucleus of the cells was stained with PI, a red nuclear counterstain, whereas the a-SMA positive cells were stained in green, which is conferred by the FITC conjugated secondary antibody. Confocal microscope at 200x magnification. Bar in the corner of each image represents $50 \mu \mathrm{m}$. (E) $\alpha$-SMA mRNA levels in cells derived from non-PD TA tissue and PD plaque tissue exposed to $10 \mathrm{ng} / \mathrm{ml}$ of TGF- $\beta 1$ for 72 hours. (F) Ratio of $\alpha$-SMA positive cells in cells derived from non-PD TA tissue and PD plaque tissue exposed to TGF- $\beta 1$ at $10 \mathrm{ng} / \mathrm{ml}$ for 72 hours, after which, the number of myofibroblasts was calculated by counting the number of $\alpha$-SMA positive cells in each field and then divided by the number of total cells. Data points were plotted as mean $\pm S E M, N=3$. Legend: * indicates $\mathrm{P}<0.05$ tested by Student's t-test vs untreated cells.

Figure 2: $\alpha$-SMA protein levels in cells derived from non-PD TA tissue and PD plaque tissue. In-Cell ELISA was performed to assess the $\alpha$-SMA protein levels in fibroblasts exposed to TGF- $\beta 1$ at $10 \mathrm{ng} / \mathrm{ml}$ for 72 hours. Data points were plotted as mean \pm SEM, $N=3$. Legend: * indicates $P<0.05$ tested by Student's t-test vs untreated cells.

Figure 3: Adenosine receptors mRNA levels in cells derived from non-PD TA tissue and PD plaque tissue. The expression of adenosine receptors was determined using the $2^{\Delta C q}$ method, where the result obtained corresponds to the fold change of each adenosine receptor relative to the expression of reference genes (EIF4A2 and TOP1). Each sample was run in triplicate. Data points were plotted as mean \pm SEM, $\mathrm{N}=3$. Legend: * indicates $\mathrm{P}<0.05$ tested by Student's t-test vs non-PD TA cells.

Figure 4: Expression of ADORA1 in cells derived from non-PD TA tissue and PD plaque tissue. (A) ADORA1 mRNA levels and (B) ADORA1 protein levels. Fibroblasts were treated with TGF- $\beta 1 \mathrm{at} 10 \mathrm{ng} / \mathrm{ml}$ for 72 hours. Data points were plotted as mean \pm SEM, $N=3$. Legend: * indicates $P<0.05$ tested by Student's t-test vs untreated cells.

Figure 5: Expression of ADORA2B in cells derived from non-PD TA tissue and PD plaque tissue. (A) $A D O R A 2 B$ mRNA levels and (B) ADORA2B protein levels. Fibroblasts were treated with TGF- $\beta 1$ at 10 $\mathrm{ng} / \mathrm{ml}$ for 72 hours. Data points were plotted as mean \pm SEM, $\mathrm{N}=3$. Legend: * indicates $\mathrm{P}<0.05$ tested by Student's t-test vs untreated cells and \# indicates $P<0.05$ tested by Student's t-test vs non-PD TA cells. 
Figure 6: Effect of adenosine receptor ligands on TGF- $\beta 1$-induced myofibroblast transformation. (A) Effect of CPA (ADORA1 agonist), (B) BAY 60-6583 (ADORA2B agonist), (C) SLV 320 (ADORA1 antagonist) and (D) MRS 1754 (ADORA2B antagonist) on TGF- $\beta 1$-induced myofibroblast transformation and cell viability. Cells derived from non-PD TA tissue and PD plaque tissue were exposed to a range of concentrations of the ligands between 0.03 and $100 \mu \mathrm{M}$ and applied in co-incubation with TGF- $\beta 1$ for 72 hours. Data points were plotted as average \pm SEM of the percentage of maximum response of either the a-SMA/DNA staining ratio (measure of myofibroblast transformation) or DNA staining (measure of cell numbers and viability), $N=3$. (E) Representative images of individual wells in an ICE plate obtained from Odyssey infrared imager in the presence of TGF- $\beta 1$ and increasing concentrations of BAY 60-6583.

Supplementary Figure 1: Effect of ADORA2B agonist, BAY 60-6583 on unstimulated fibroblasts. Cells derived from non-PD TA tissue and PD plaque tissue were exposed BAY 60-6583 (0.03 - $100 \mu \mathrm{M})$ for 72 hours. Data points were plotted as average \pm SEM of the percentage of maximum response of either the $\alpha$-SMA/DNA staining ratio (measure of myofibroblast transformation) or DNA staining (measure of cell numbers and viability), $N=3$. Please note that no $\alpha-S M A$ was observed since these cells were not exposed to TGF- $\beta 1$. 


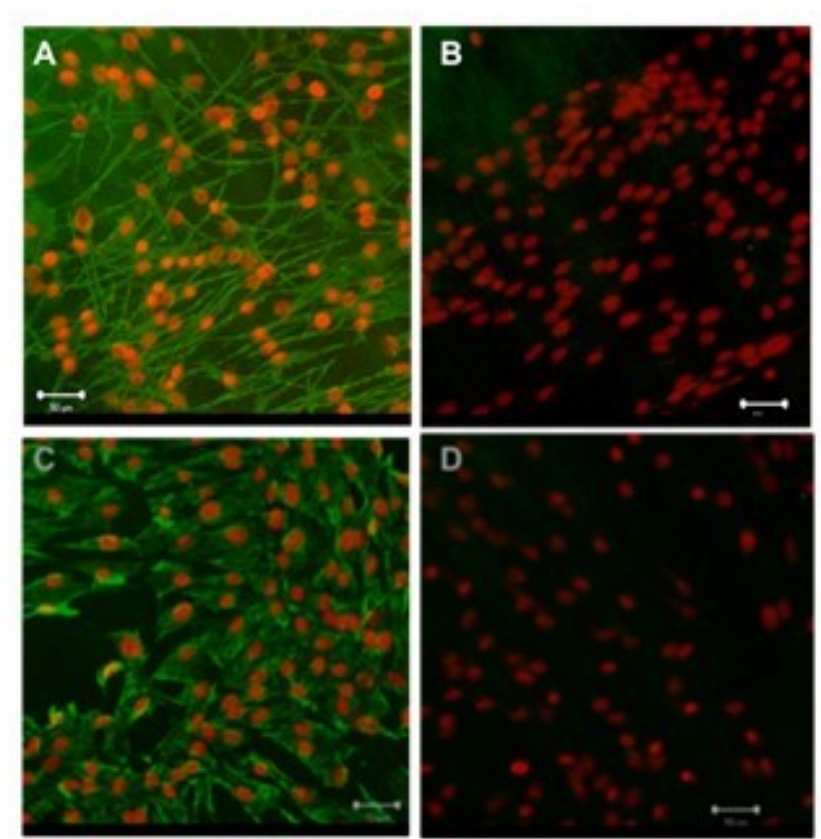

G

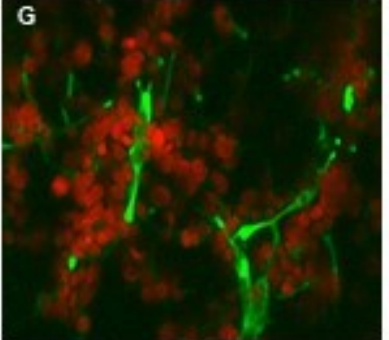

H

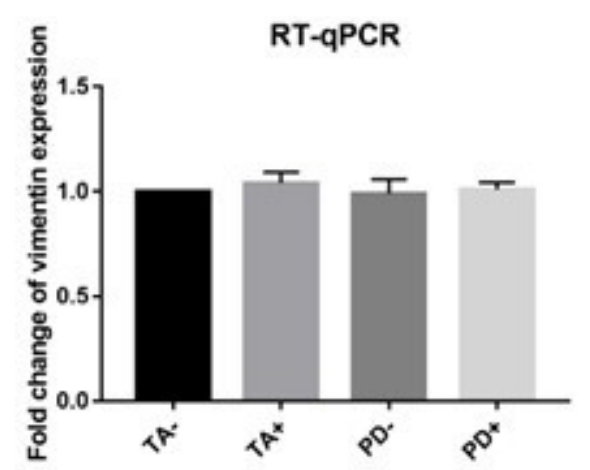

Non-PD TA cells

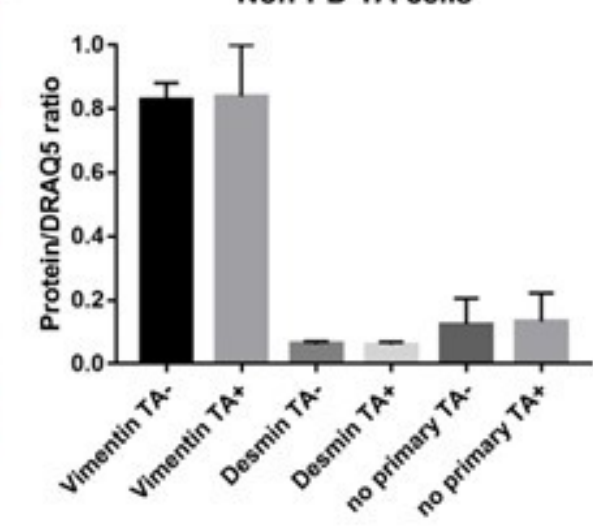

PD TA cells
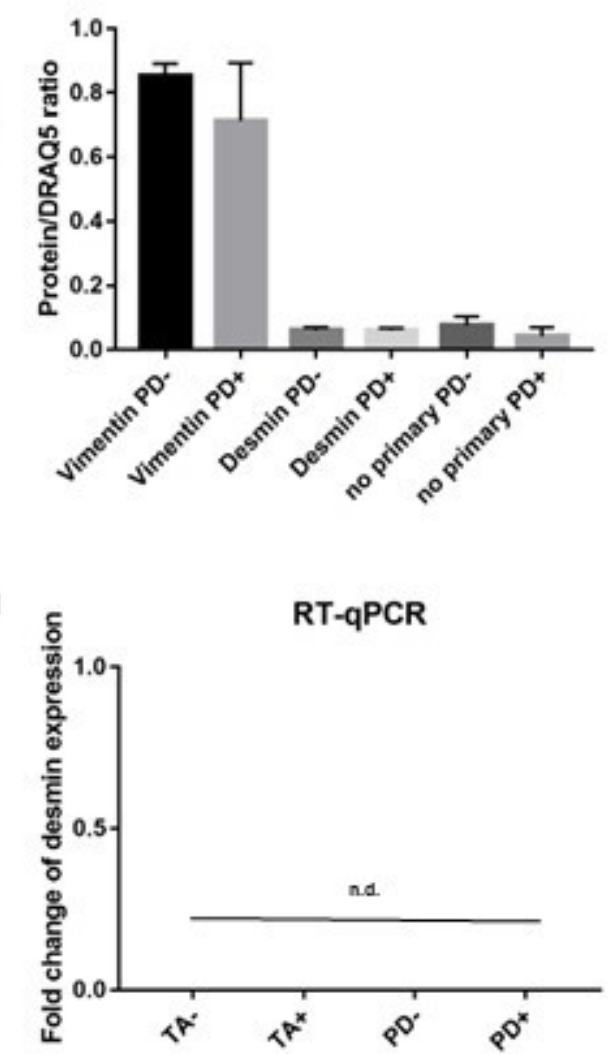

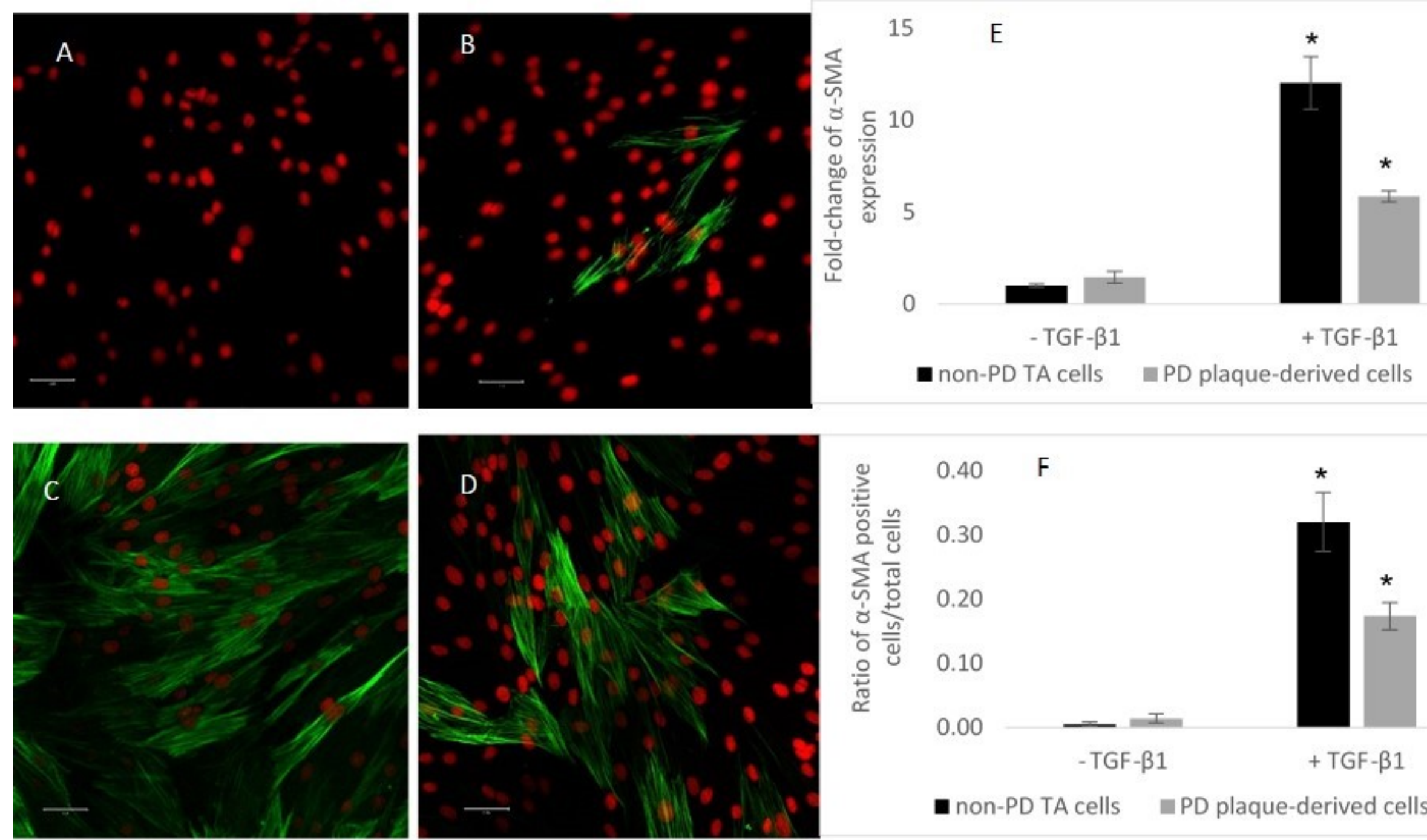

F

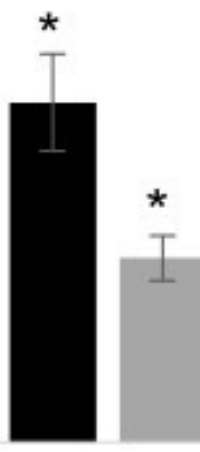

+ TGF- $\beta 1$

anon-PD TA cells a PD plaque-derived cells 


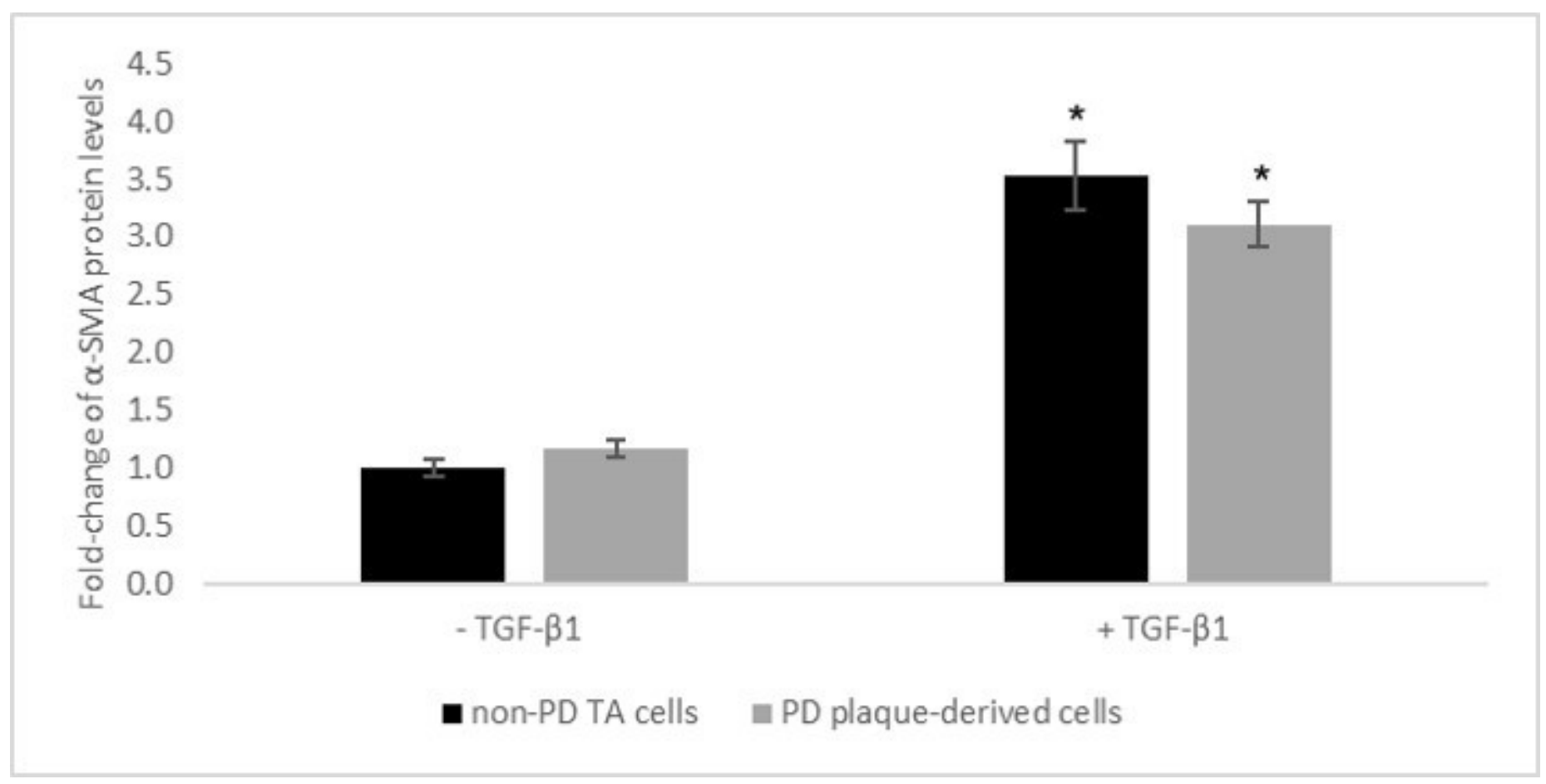




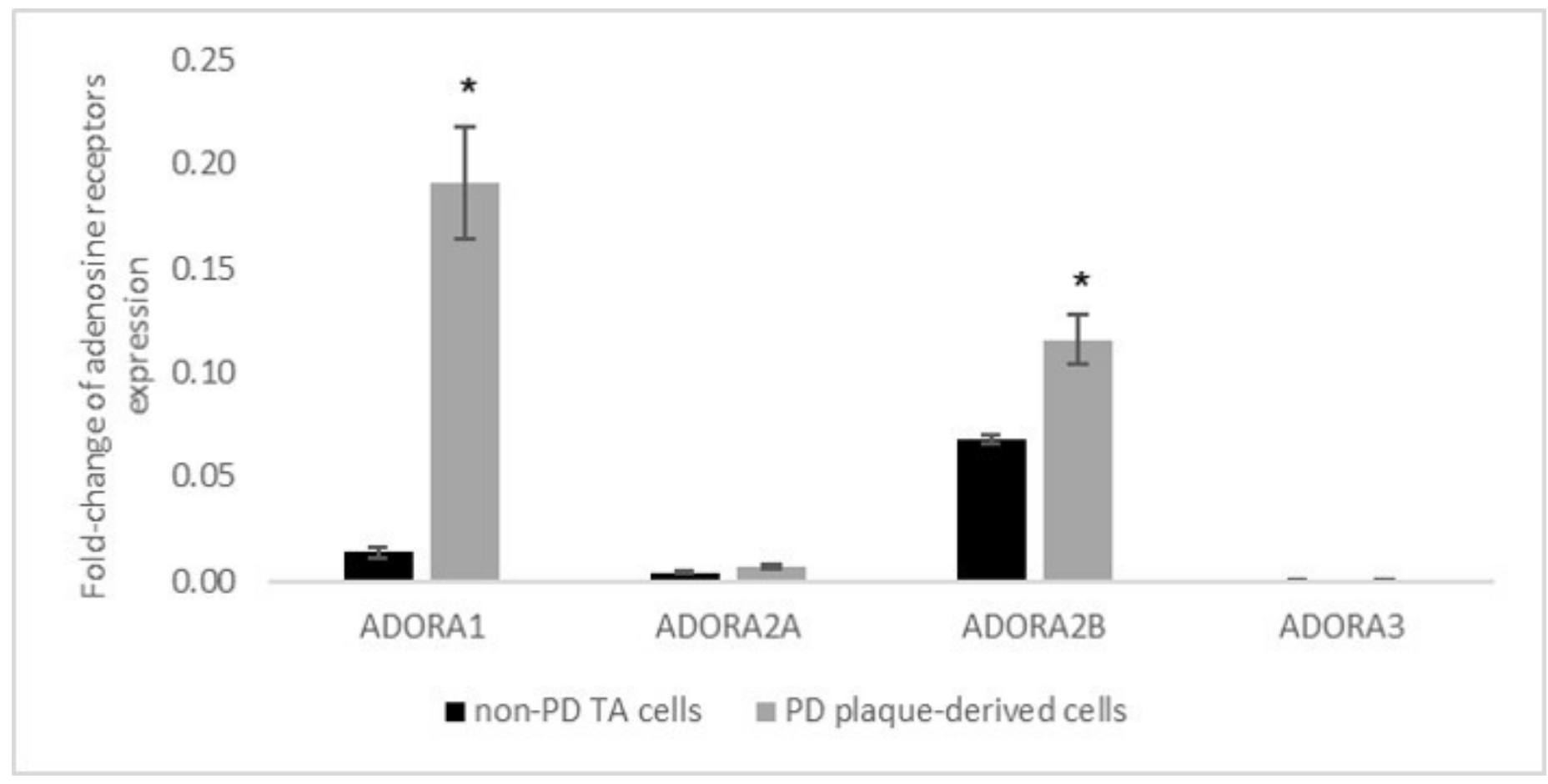




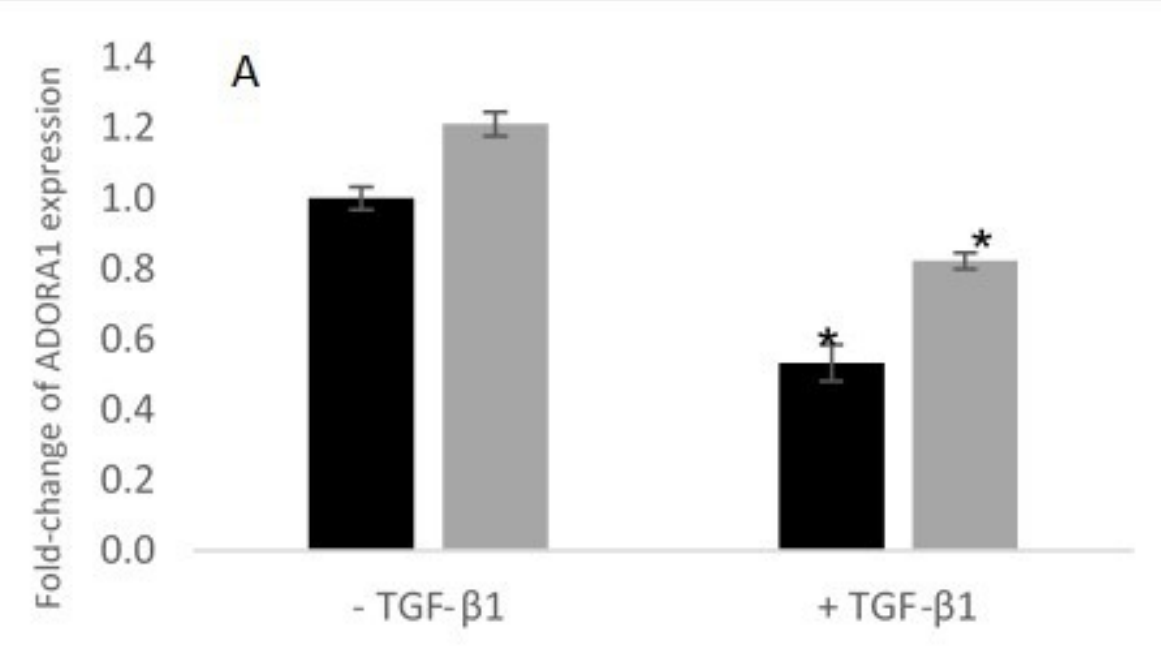

- non-PD TA cells a PD plaque-derived cells

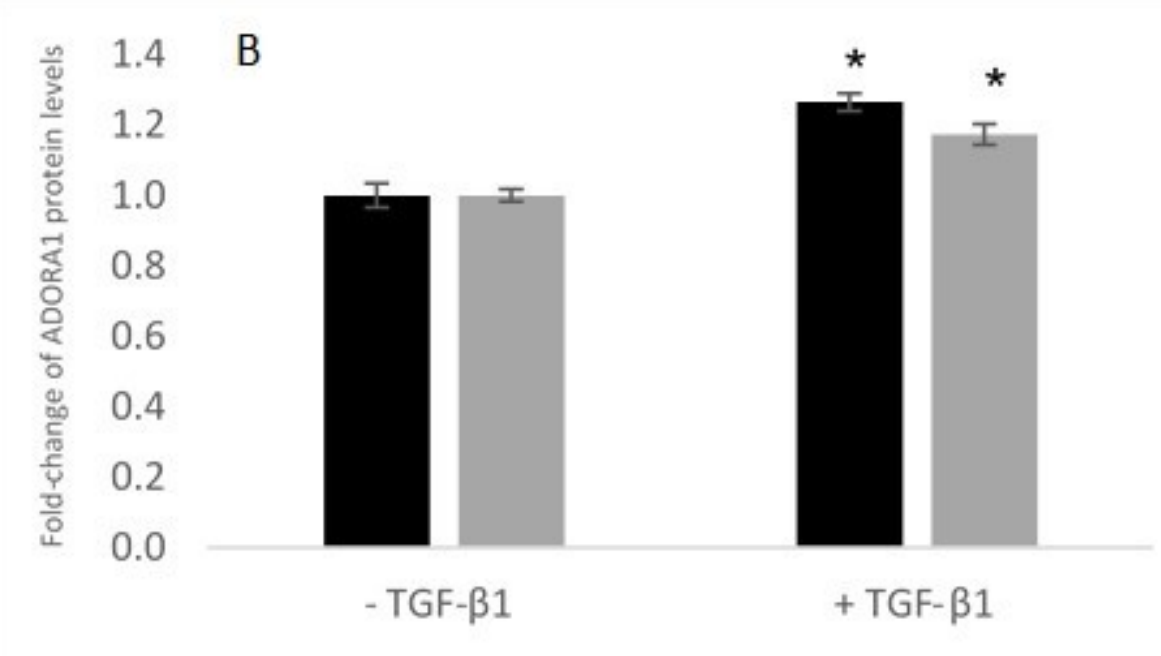

- non-PD TA cells aPD plaque-derived cells 


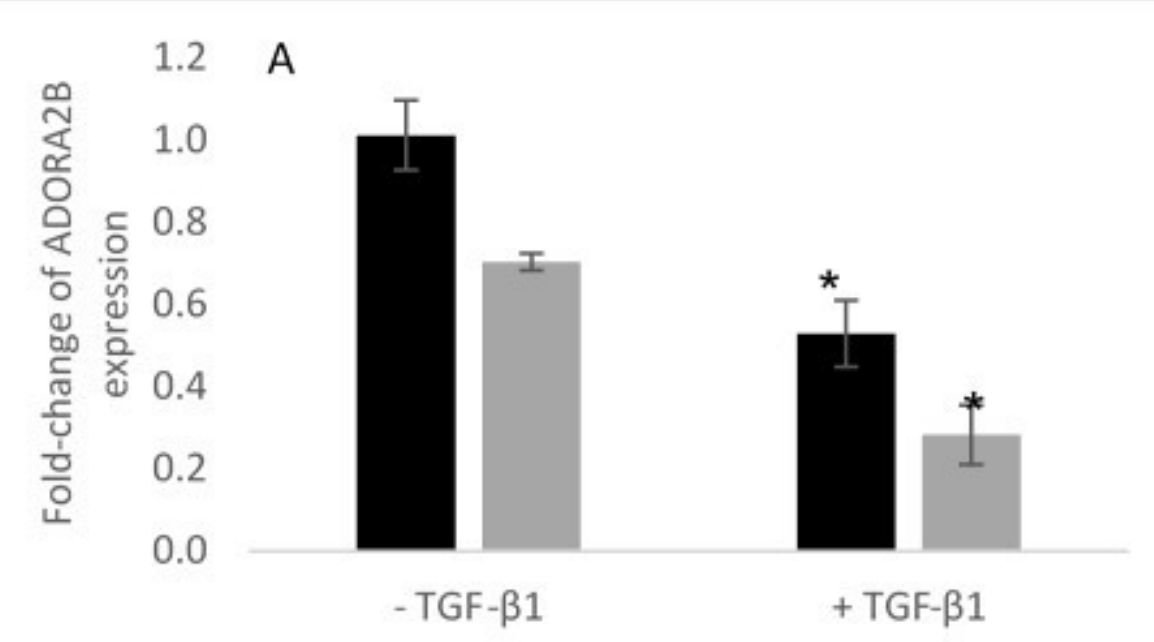

- non-PD TA cells

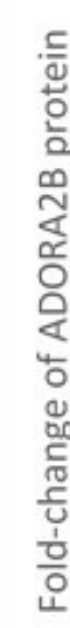

2.5

2.0

\#

\#

\#

㓂

ㅇํㅇำ 1.5

造 1.0

○

0.5

0.5
0.0
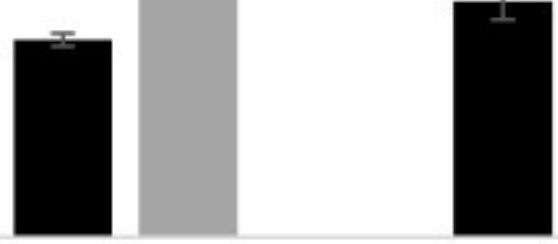

- TGF- $\beta 1$

$+\mathrm{TGF}-\beta 1$

non-PD TA cells a PD plaque-derived cells 


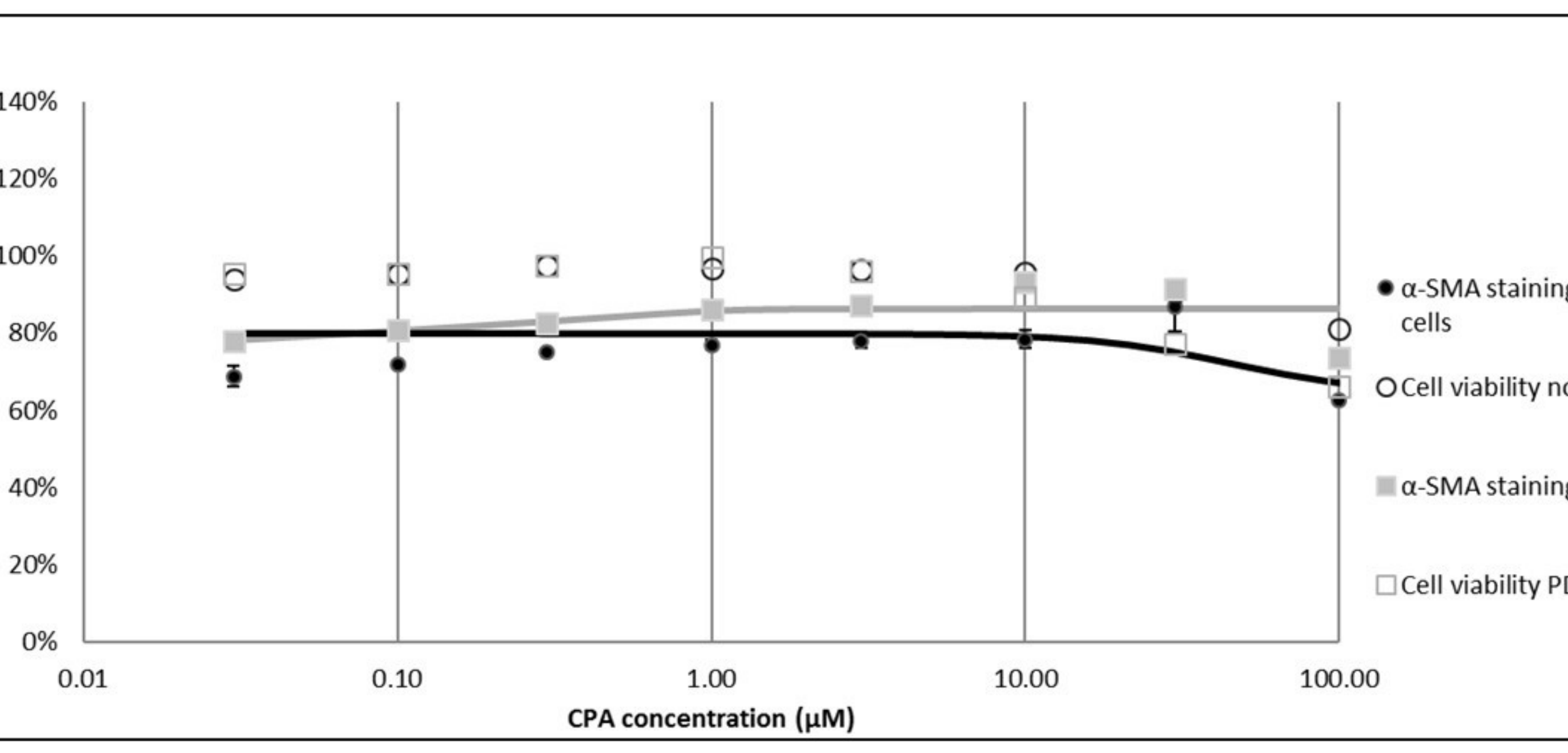




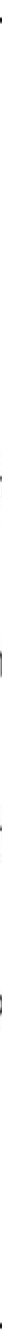




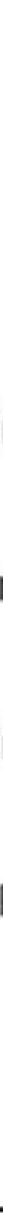


E

BAY 60-6583 $(\mu \mathrm{M})$

$\begin{array}{llllllll}0.03 & 0.1 & 0.3 & 1 & 3 & 10 & 30 & 100\end{array}$

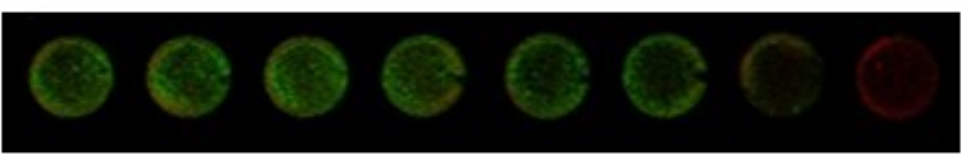




\section{Supplementary Figure 1}

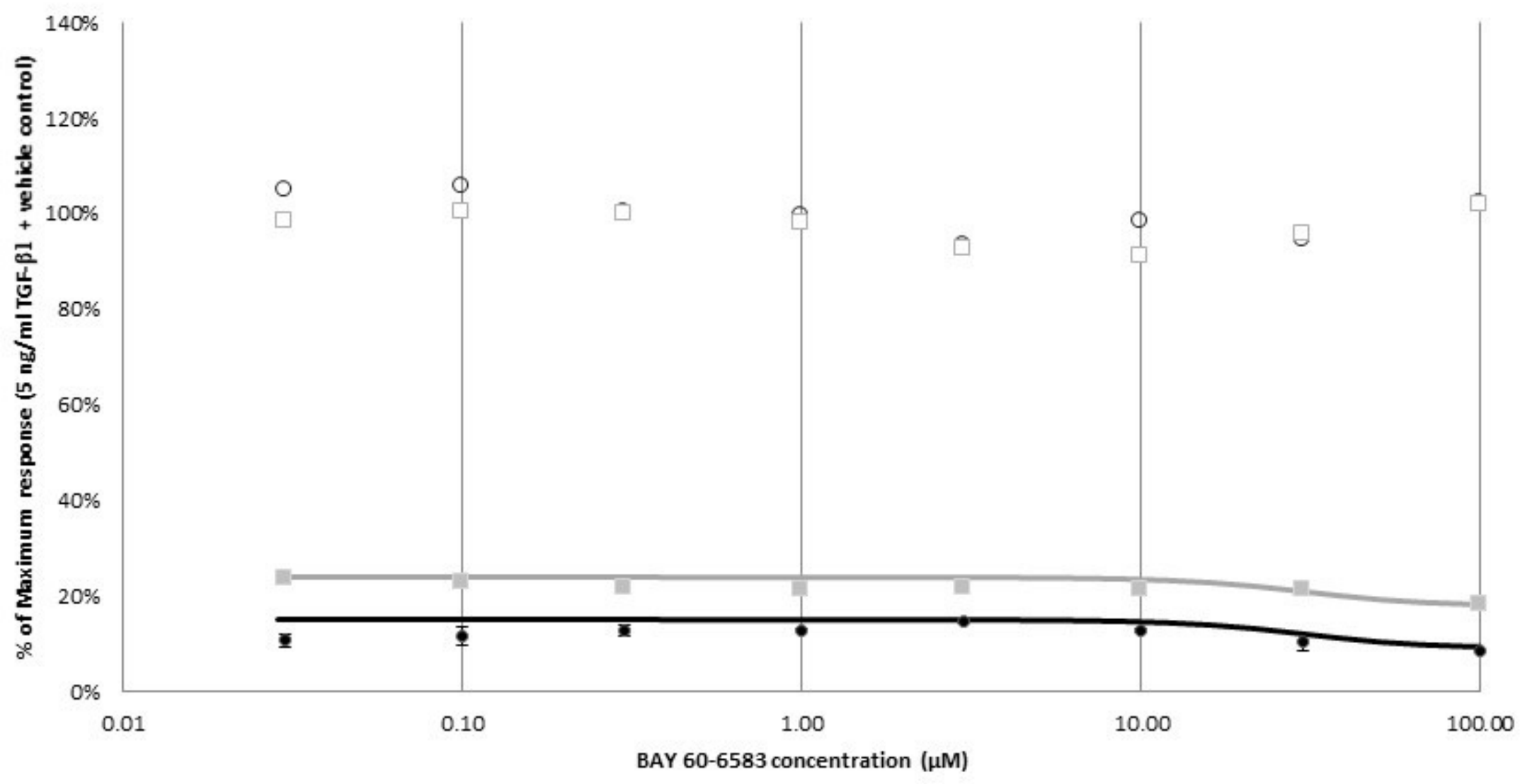

- $\alpha$-SMA staining non-PD ce

O Cell viability non-PD cells

$\alpha$-SMA staining PD cells

$\square$ Cell viability PD cells 